Article

\title{
Interfacial and Bending Properties by Adding HNTs on the Aramid/Basalt Reinforced Epoxy-Based Hybrid Composites
}

\author{
Jin-Woo Lee ${ }^{1}$, Tianyu Yu ${ }^{2}$, Chang-Wook Park ${ }^{2}$ and Yun-Hae Kim 1,* \\ 1 Department of Mechanical Engineering, Korea Maritime and Ocean University, 49112 Busan, Korea; \\ zenith1179@naver.com \\ 2 Department of Material Engineering, Graduate School, Korea Maritime and Ocean University, \\ 49112 Busan, Korea; pcw0591@naver.com \\ * Correspondence: yunheak@kmou.ac.kr; Tel.: +82-51-410-4966
}

\begin{abstract}
Hybrid fiber reinforced composites can be controlled by price, weight and various mechanical properties depending on fiber ratio and lamination method. Despite these excellent hybrid properties, there is a disadvantage that inter-laminar fracture due to external impact, which is the biggest weakness of fiber reinforced composite materials, is weak. The test specimens were prepared by using a vacuum bag method, which is manufactured by using an autoclave device. The pre-preg is manufactured in the form of a B-stage. In the process of fabricating the nanoparticle prepreg, the homogeneizer using an ultrasonic wave was used to disperse the epoxy subject without the curing agent into nanoparticles. The dispersion of the nanoparticles was dispersed by the weight of the epoxy resin. This is to take into account the cohesion of HNT and to understand the range of cohesion of HNT in a matrix with viscosity and its phenomenon. According to the Comparison of the interlayer interfacial properties and mechanical properties of Aramid / Basalt fiber hybrid composites by HNT addition, the fracture toughness, ILSS and bending strength of specimens with HNT content of more than a certain level were decreased because of the aggregation of HNT.
\end{abstract}

Keywords: halloysite nanotube(HNT); hybrid composite; aramid fiber; basalt fiber; interfacial property; fiber reinforced composite; aggregation

\section{Introduction}

Hybrid fiber reinforced composites are composed of two or more reinforcing fibers and matrix materials. Therefore, it is possible to improve the performance of the fiber reinforced composite by the hybrid effect which improves the disadvantages of the other fibers by mixing different reinforcing fibers and emphasizes the advantages [1-2]. Hybrid fiber reinforced composites can be controlled by price, weight and various mechanical properties depending on fiber ratio and lamination method. Despite these excellent hybrid properties, there is a disadvantage that inter-laminar fracture due to external impact, which is the biggest weakness of fiber reinforced composite materials, is weak. Particularly, hybrid composite materials using heterogeneous fibers have a considerable effect of interlayer separation between different fibers, which is a major cause of decrease in the strength [3].

Fracture behavior of laminated composites can be classified into three types. The first is a trans-laminar fracture, which means that cracks have progressed in the direction of cutting the fibers. The transverse fracture does not occur singly but is locally observed along with the following two types of fracture. The second is intra-laminar, which means cracks propagate along the fiber direction. This fracture pattern is often seen when first ply failure or the weakest layer first breaks. The third is delamination in which inter-laminar separation occurs between layers. Since the fiber of the highgrade composite material is much stronger in strength than the matrix, the macroscopic fracture generally occurs after the inner layer fracture and the interlayer fracture are sufficiently grown in combination, and the lateral fracture occurs after reaching the final fracture [4]. 
The most common lifetime limit damage mode for laminated composites is delamination. The interlayer separation grows in the base layer between the layers. In general structures, delamination occurs due to static or fatigue loading during manufacture or use of actual structures, where stress concentration occurs. This phenomenon causes the rigidity and strength of the material to be lowered and causes a sudden breakage of the structure. Therefore, obtaining a reliable value of the interlaminar separation resistance of the fiber-reinforced composite material is the most important part in designing the structure of the composite material, and studies on the mode $\Pi$ critical energy release rate for explaining the destruction phenomenon of the layer shortness are actively conducted [5].

Generally, it is known that a laminate type composite material to which no particles are added exhibits a low inter-laminar shear strength (ILSS) or a critical stress intensity factor (KIC) is poor in wettability with the matrix and the surface itself is smooth so that the interface bonding force is weak. In order to improve the interfacial bonding strength of carbon fiber reinforced composite materials, it is necessary to remove impurities on the surface of carbon fiber by a surface treatment such as gas phase oxidation, liquid phase oxidation, anodic oxidation, and plasma And hydroxyl to enhance the interfacial bonding between carbon fibers and matrix, and to improve interfacial bonding by adding other small amounts of materials such as $\mathrm{SiC}$ and $\mathrm{TiO}_{2}$ between carbon fibers and matrix [6-9].

Therefore, in this study, we investigated the mechanical properties and fracture toughness of hybrid fiber reinforced composites by measuring bending strength and ILSS by adding HNT, which is considered to be a weak point of hybrid fiber reinforced composites.

\section{Experimental Procedures}

\subsection{Manufacture of Specimens}

In the experiment, woven fabric with plain weaving at $0^{\circ}$ and $90^{\circ}$ aramid fiber(HF-200) and basalt fiber(HB-200), which produced by GM COMPOSITE CO. Ltd (Korea), are used as the reinforcement and epoxy resin (KFR-120) are used as the matrix and in this experiment, $28 \mathrm{wt} . \%$ hardener (KFH-141) was mixed with resin to form the matrix. The specification of reinforcement and matrix are shown in table 1 and table 2 .

In each case, the HNT content was dispersed in the base material under the same ultrasonic dispersion conditions and then molded. The reason for using ultrasonic phase separation device is intended to combine the characteristics of the phenomenon of cavitation ultrasonic waves in the composite material. The test specimens were prepared by using a vacuum bag method, which is manufactured by using an autoclave device, which has high mechanical properties and high reliability of physical properties. The curing conditions used in the fabrication are shown in Figure 1. The pre-preg is manufactured in the form of a B-stage in which the resin is previously impregnated with the resin to complete the curing reaction. Thus, even if the pre-preg of the nanoparticles used in this study is not sold in the market, And then used. In the process of fabricating the nanoparticle prepreg, the homogeneizer using an ultrasonic wave was used to disperse the epoxy subject without the curing agent into nanoparticles. The dispersion of the nanoparticles was dispersed by the weight of the epoxy resin. The content of HNT was limited to $0,0.5,1,2,3 \mathrm{wt} \%$. This is to take into account the cohesion of HNT and to understand the range of cohesion of HNT in a matrix with viscosity and its phenomenon.

Table 1. Specification of aramid and basalt plain woven fabric.

\begin{tabular}{ccc}
\hline & Aramid(HF-200) & Basalt(HB-200) \\
\hline Fabric weight(g/m2) & 165 & $200 \pm 16$ \\
Warp construction(count/in) & 13 & 7.5 \\
Fill construction(count/in) & 13 & 7.5 \\
Fab thickness(mm) & $0.18 \pm 0.025$ & $0.18 \pm 0.025$ \\
\hline
\end{tabular}


Table 2. Properties of epoxy resin (KFR-120).

\begin{tabular}{cc}
\hline Property & Detail \\
\hline Density $(\mathrm{g} / \mathrm{m} 3)$ & $1.0 \sim 1.2$ \\
Tensile strength $(\mathrm{MPa})$ & $70 \sim 80$ \\
Elastic modulus(GPa) & $3.1 \sim 3.3$ \\
Elongation at break $(\%)$ & $7.9 \sim 8.1$ \\
\hline
\end{tabular}

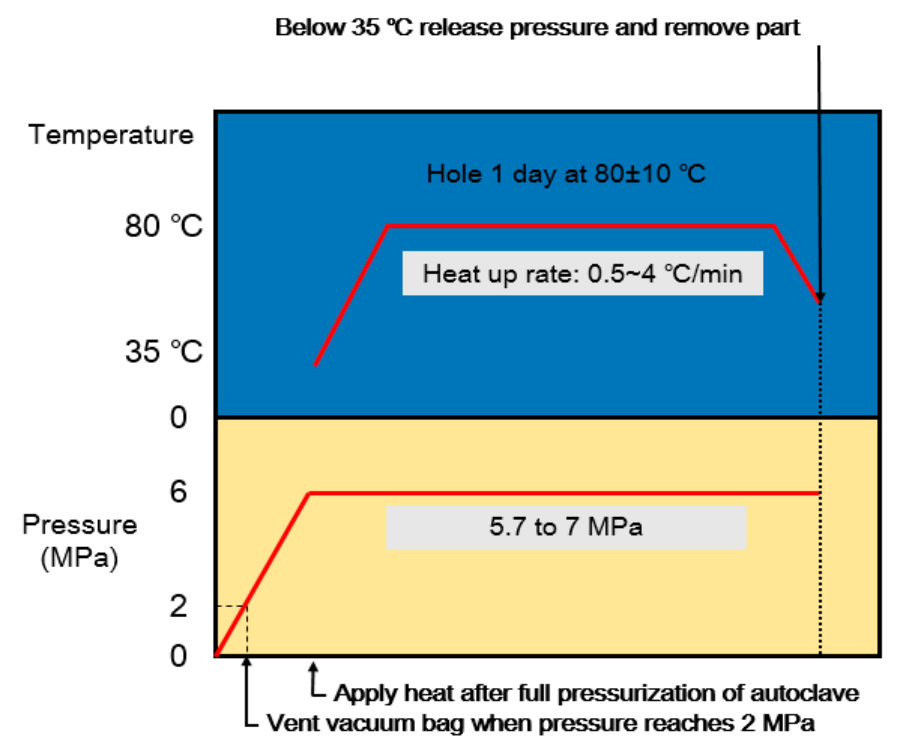

Figure 1. Curing conditions for specimen preparation.

\subsection{Evaluation method of mechanical and interface properties}

6 samples with different aramid/basalt fiber ply number are made to find the relation of mechanical properties with different fiber ratio. In this situation, samples all have laminates of 14 sheets and changes with aramid/basalt ply number: $0 / 14,2 / 12,4 / 10,6 / 8,8 / 6,10 / 4,12 / 2,14 / 0$. All basalt plies are in the middle of the sample and the aramid plies are put on the both sides of basalt ply averagely. 6 samples with different ply sequence are also produced, the total ply number is 14 sheets invariably and 7 aramid sheets 7 basalt sheets are used in every sample. Every stacking sequence was considered to have different aramid/basalt interface number and be maximum approximately symmetrical. The details of ply sequence and laminate codes are shown in table. 4 .

Table 3. List of the prepared laminates with stacking sequence.

\begin{tabular}{ccc}
\hline Laminate code & Number of laminate & Laminating structure \\
\hline AFRP & 14 & {$[\mathrm{~A}] 20$} \\
BFRP & 14 & {$[\mathrm{~B}] 20$} \\
A2B12 & $2(\mathrm{~A})+12(\mathrm{~B})$ & ABBBBBBBBBBBBA \\
A4B10 & $4(\mathrm{~A})+10(\mathrm{~B})$ & AABBBBBBBBBBAA \\
A6B8 & $6(\mathrm{~A})+8(\mathrm{~B})$ & AAABBBBBBBBAAA \\
A8B6 & $8(\mathrm{~A})+6(\mathrm{~B})$ & AAAABBBBBBAAAA \\
A10B4 & $10(\mathrm{~A})+4(\mathrm{~B})$ & AAAAABBBBAAAAA \\
A12B2 & $12(\mathrm{~A})+2(\mathrm{~B})$ & AAAAAABBAAAAAA \\
A & $7(\mathrm{~A})+7(\mathrm{~B})$ & ABABABABABABAB \\
B & $7(\mathrm{~A})+7(\mathrm{~B})$ & AABBAABBAABBAB \\
C & $7(\mathrm{~A})+7(\mathrm{~B})$ & AAABBBBAAAABBB \\
D & $7(\mathrm{~A})+7(\mathrm{~B})$ & AAABBBBBBBAAAA \\
E & $7(\mathrm{~A})+7(\mathrm{~B})$ & BBBAAAAAAABBBB \\
F & $7(\mathrm{~A})+7(\mathrm{~B})$ & AAAAAAABBBBBBB \\
& $\mathrm{A}=$ aramid fabric, B=basalt fabric & \\
\hline
\end{tabular}


The test specimens were measured using standard test methods for the open-hole tensile strength of polymer matrix composite laminates of ASTM D 5766 for mechanical properties evaluation. The reason for choosing the specimens with perforated notches is that the dog-bone specimens with curved surfaces can only be machined with waterjet processing and are expensive to produce. In other words, it is difficult to ensure the reliability of mechanical properties when machining. Therefore, in this study, the tensile strength was determined by measuring the test specimen with a notched hole. Further, the interlayer shear strength was measured using the ASTM D 2344 Standard test method for the short-beam strength of polymer matrix composite materials and their laminates. The DCB test specimens were prepared by laminating pre-pregs made of aramid fibers when laminating pre-pregs with nanoparticles added, adding Teflon sheets, and laminating the remaining pre-pregs to form the first cracks. The test method of DCB test specimens was conducted according to Standard Test Method for Mixed Mode I-Mode II Inter-laminar Fracture Toughness of Unidirectional Fiber Reinforced Polymer Matrix Composites (ASTM D 6671). The schematic diagram of the measurement test is shown in Figure 2.

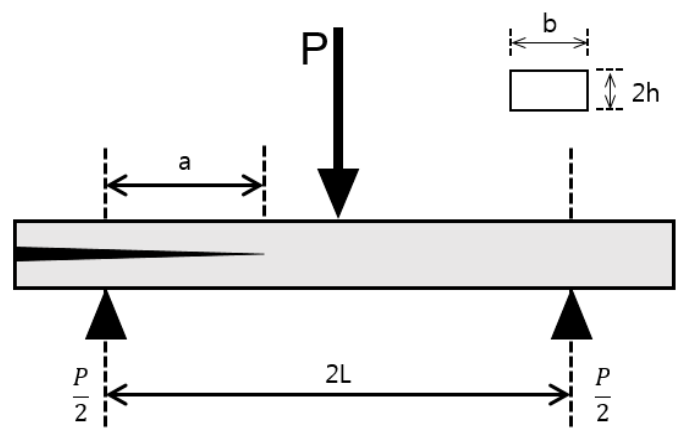

(a) geometry

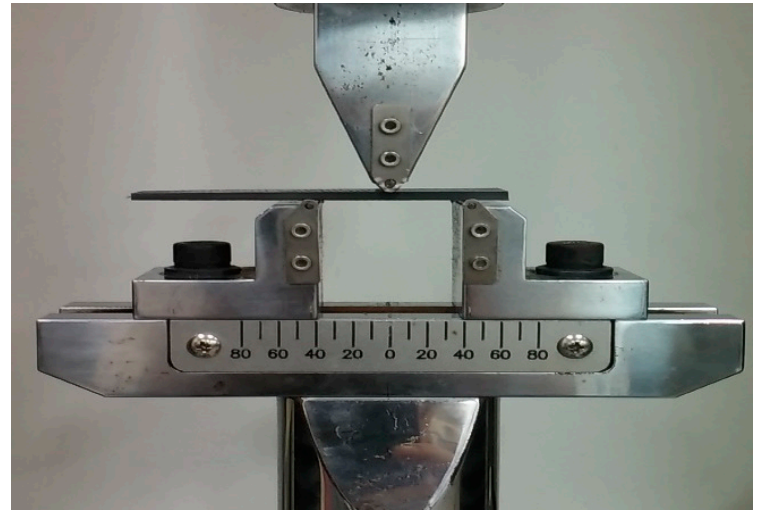

(b) Picture of mode IItest

Figure 2. Specimen geometry (mode $\Pi$ ).

\section{Results and Discussion}

\subsection{Change of bending characteristics according to lamination pattern of hybrid composite}

According to our previous research, the laminate pattern of the hybrid fiber-reinforced composite material has a great influence on the mechanical properties of the composite material [10]. Previous studies have shown that hybrid fibers of carbon fiber and basalt fiber are different from those of this study. Therefore, we investigated the bending properties of aramid / basalt hybrid composites in various patterns to find the optimal pattern to be used in the study. Figure 3-(a) is a graph of bending characteristics according to the content of aramid fibers. In general, aramid fibers are known to have better bending properties than basalt fibers. Therefore, the strength of aramid fibers tended to increase with increasing fiber content. However, it was found that the bending strength decreased from the test specimen having a content ratio of about $70 \%$ at which the bending strength showed the maximum value. It was found that the hybridization increases the bending strength by increasing the bending strength when hybridized than the bending strength of each of the basalt fiber and aramid fiber.

Figure 3-(b) is a graph of bending strength of the basalt / aramid hybrid fiber reinforced composite according to the lamination pattern. The laminate pattern showed the difference in bending strength and Laminate code $\mathrm{D}$ showed the highest bending strength. It seems that the difference between the properties of the basalt fiber and the aramid fiber affects the fiber and fiber. Thus it was determined the lamination pattern in the Laminate code D with the highest bending strength to grasp the characteristics of the fibers and the fibers. A test piece was added HNT by using this pattern was the experiment on the effect of the HNT. 


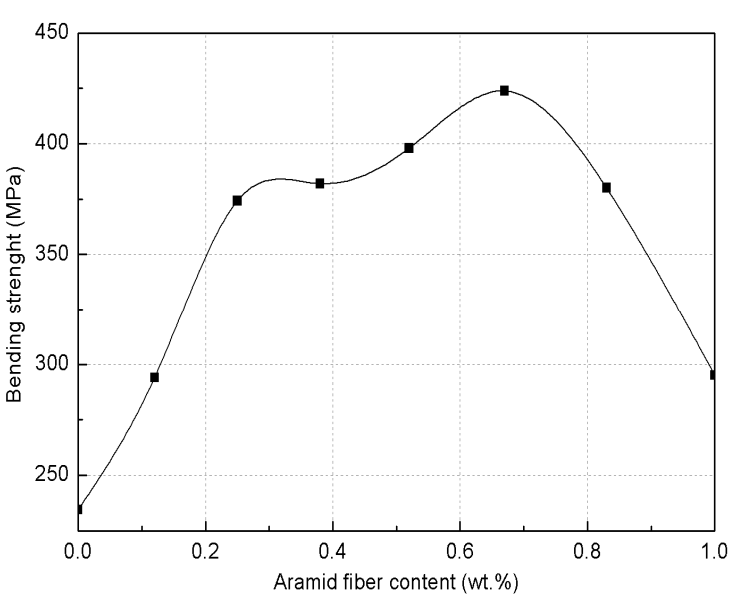

(a) aramid fiber contents

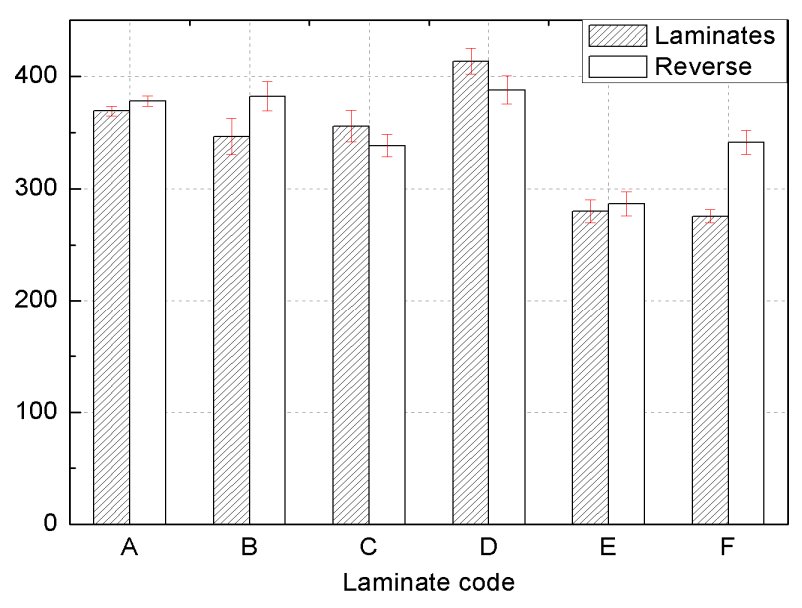

(b) ply sequence

Figure 3. Effect of Aramid Fiber Content(a) and ply sequence(b) on Bending Strength of Basalt / Aramid Hybrid Composites

\subsection{Evaluation of Mechanical Properties of Basalt / Aramid Hybrid Composites by Addition of HNT}

Figure 4 is an optical microscope image of a basalt / aramid composite without HNT. In most of the specimens, fiber rupture fracture occurred between the basalt fiber and the aramid fiber. Delamination was in particular a remarkably generated between basalt fibers and aramid fibers. This is considered to have occurred because of the low inter-laminar interfacial bond between the biggest weakness of two kinds of fibers of the hybrid composite material. Therefore, the change of bending strength and the interfacial inter-facial properties according to the addition amount of HNT were evaluated.
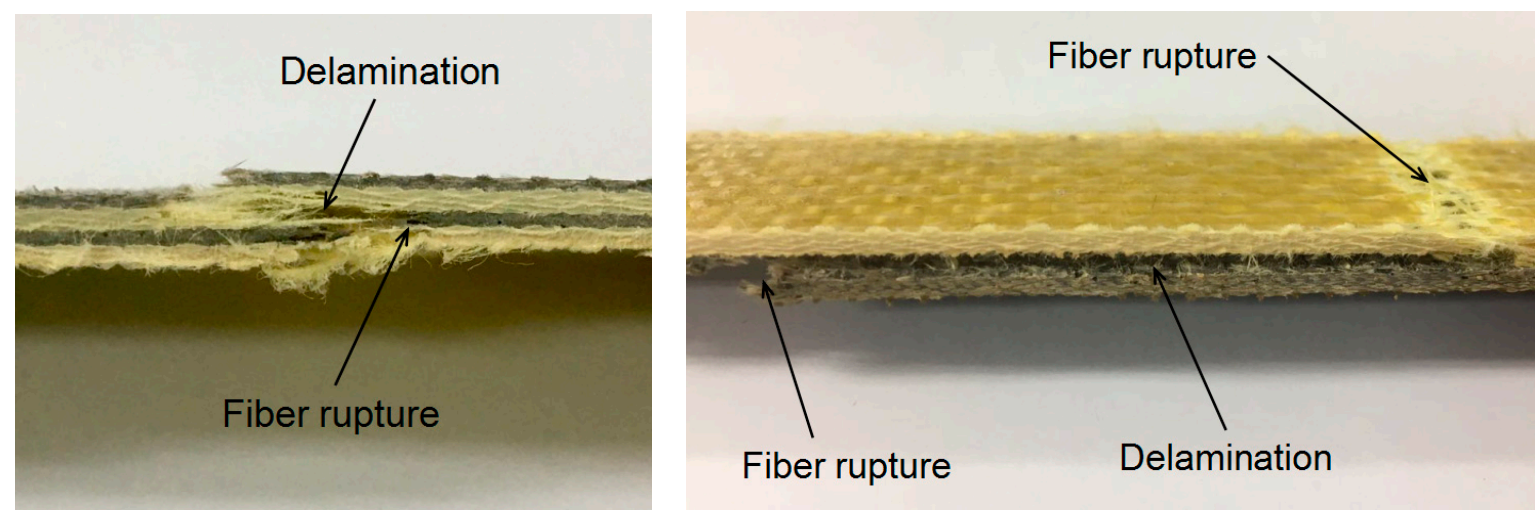

Figure 4. Microscope image of basalt / aramid hybrid composites after bending test

Figure 5-(a) is a graph showing the bending strength according to the amount of HNT added to an aramid / basalt hybrid fiber reinforced composite material. When the content was $2 \mathrm{wt} \%$, the highest bending strength was shown. HNT - reinforced hybrid composites showed bending strength reinforcement effect by HNT. However, the reinforcing effect was different depending on the contents, and it tended to increase up to $2 \mathrm{wt} \%$.

Figure 5-(b) is shown the result of the ILSS evaluated to examine the influence of the inter-layer interface by the HNT addition of basalt / aramid fiber reinforced hybrid composites. It can be seen that the reinforcing effect on the inter-laminar interface is generally observed except for the $0.5 \mathrm{wt} \%$ test piece which was lower than the hybrid composite without HNT. It is considered that can help reduce the Delamination at the interface between the interlayer weakness of the hybrid composite material. 


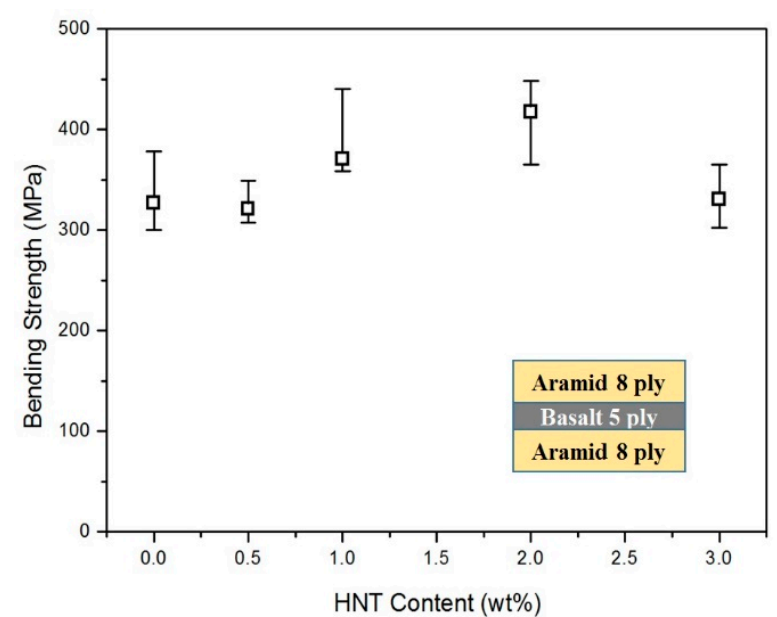

(a) bending strength

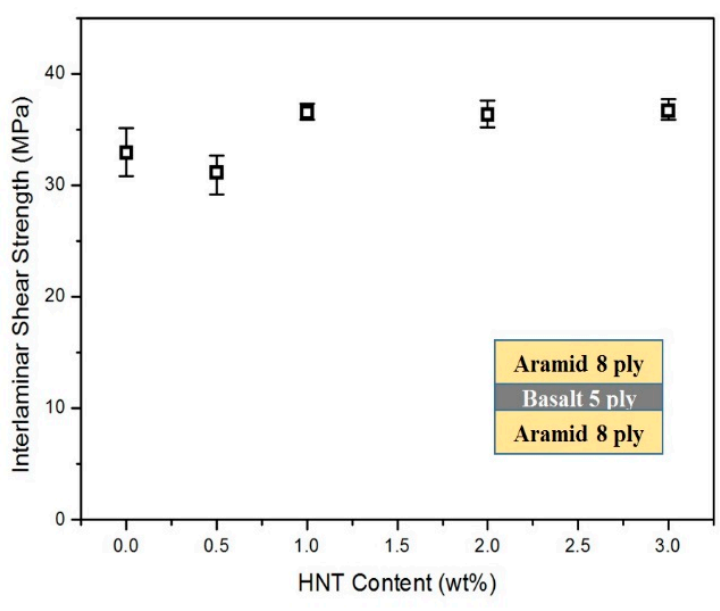

(b) interlaminar shear strength

Figure 5. Mechanical Properties of Laminated Code D Basalt / Aramid Hybrid Composites by HNT Contents

Figure 6 is a graph showing the inter-laminar fracture toughness between the basalt fiber and the aramid fiber. The fracture toughness between the two fibers was determined by inserting a Teflon sheet between the aramid fiber and the basalt fiber to obtain the interlayer interfacial properties, which are a major weakness of hybrid composites using different fibers. Due to the different elastic modulus and fiber properties of the two fibers, the fracture toughness of the hybrid composite specimens without HNT was found to be low. The addition of HNT could generally determine the reinforcing effect of the interlayer through the fracture toughness. Further, when the content of HNT of $1 \mathrm{wt} \%$ was able to confirm that it has the highest fracture toughness.

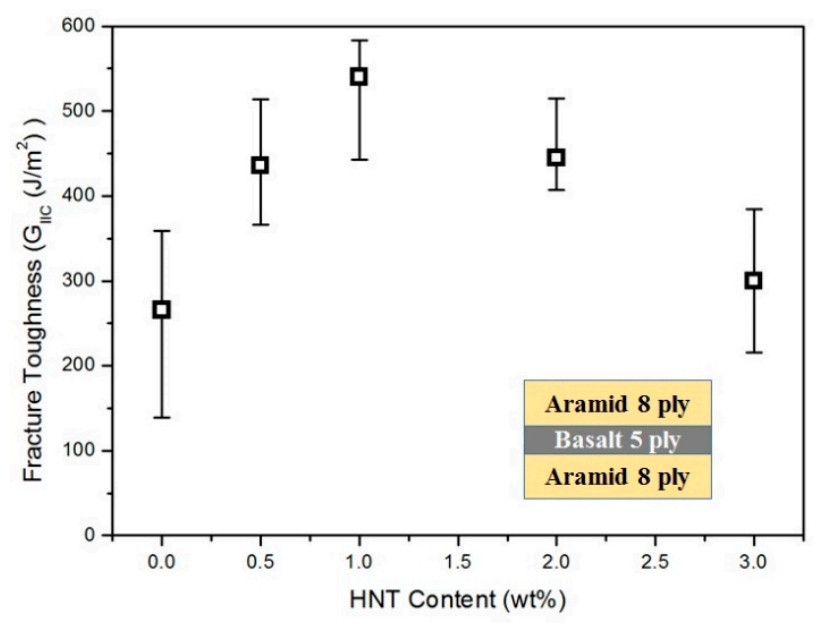

Figure 6. Fracture toughness of laminated code D Basalt / Aramid hybrid composites by HNT contents.

The bending strength, fracture toughness and ILSS of the Aramid / Basalt fiber hybrid composite are shown in the graph of Figure 7 to analyze the interlayer interfacial properties and mechanical properties of HNT. HNT content with the highest fracture toughness was $1 \mathrm{wt} \%$ hybrid composite, and the highest ILSS content was $2 \mathrm{wt} \%$ and $3 \mathrm{wt} \%$. This was different from the tendency of bending strength. This suggests that the fracture toughness is increased due to the reinforcing effect, and the overall bending strength is increased by the addition of HNT. The reason for the highest bending strength at $2 \mathrm{wt} \%$ is that the reinforcing effect of each layer is the highest at $2 \mathrm{wt} \%$ of interlayer interfacial shear strength, which is evidence of reinforcing effect to shear by addition of HNT. However, as a result of previous studies, it seems that the addition of HNT above a certain amount 
causes the HNT to coagulate due to the high surface energy of HNT, resulting in a decrease in strength. Figure 8 is an image obtained by measuring the fracture surface with SEM after bending strength test as a result of HNT aggregation. This SEM image can be used to easily identify HNT aggregation.

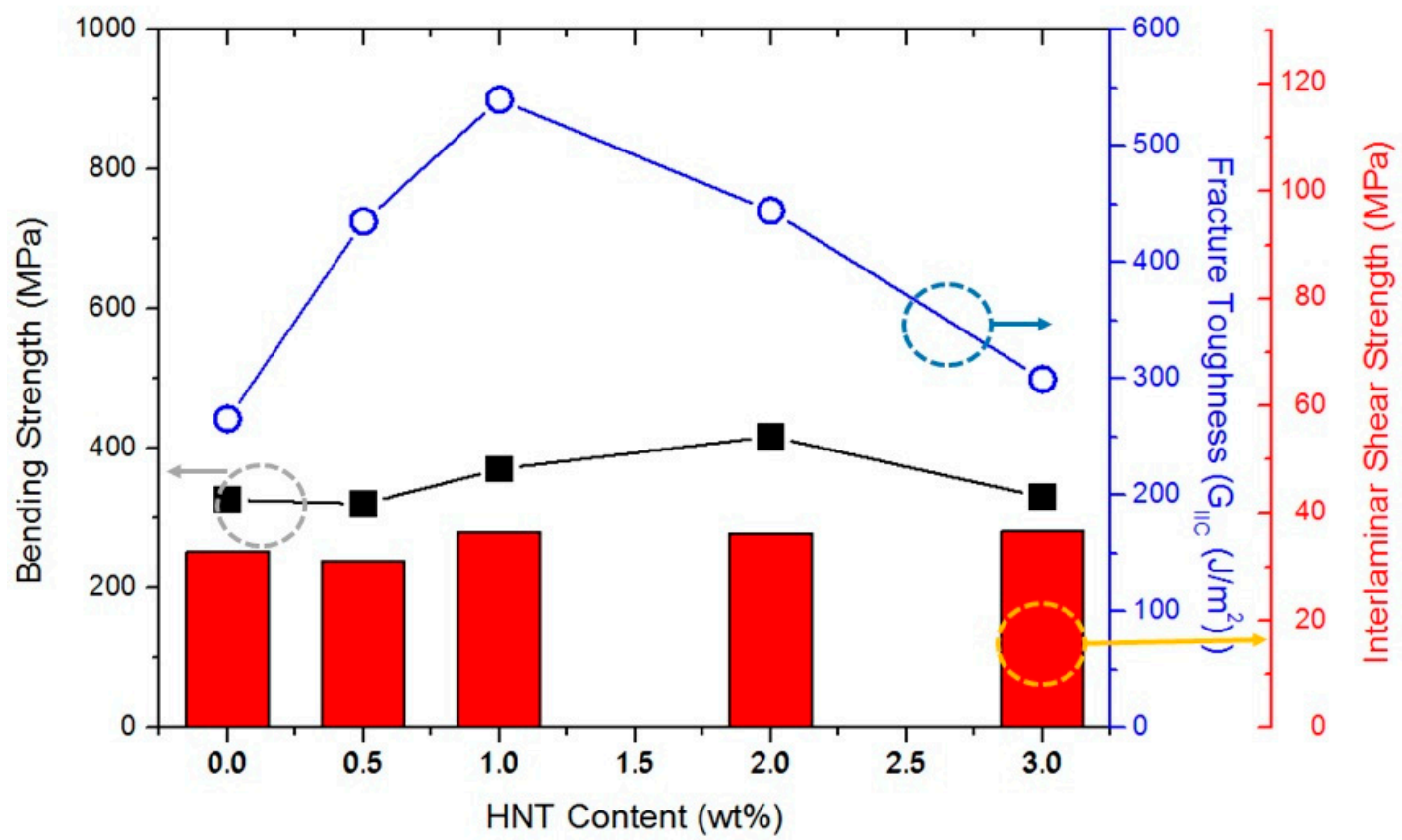

Figure 7. Comparison of the interlayer interfacial properties and mechanical properties of Aramid / Basalt fiber hybrid composites by HNT addition.

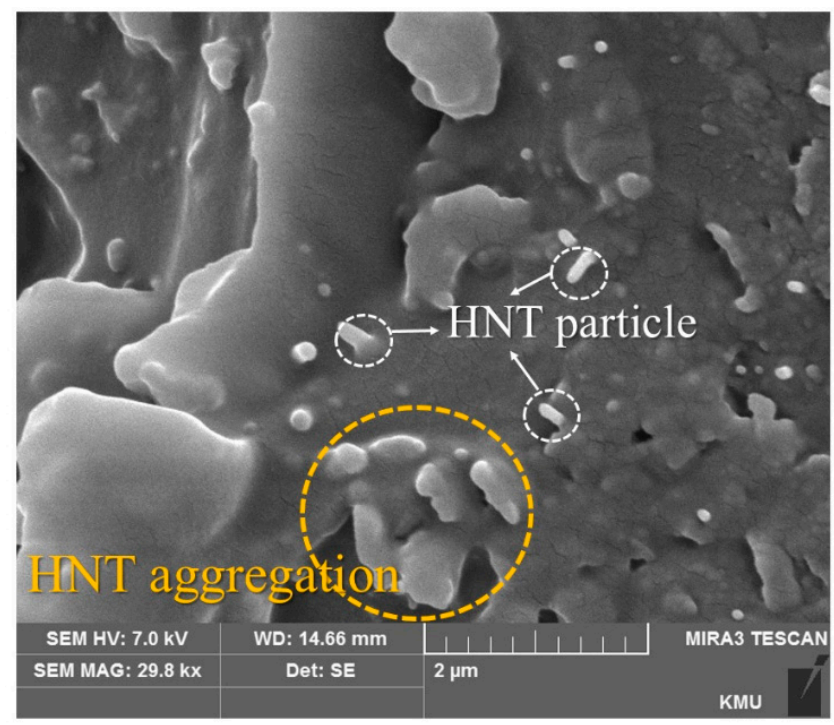

Figure 8. SEM image of $3 \mathrm{wt} \%$ HNT-added basalt / aramid hybrid composites.

\section{Conclusions}

In order to improve the bending strength and interfacial properties of the basalt fiber / aramid fiber reinforced hybrid composites, the characteristics of hybrid composites by the addition of HNT were evaluated and the following conclusions were obtained.

(1) The flexural strength of hybrid fibers obtained by mixing two types of fibers was higher than the flexural strength of composite materials composed of either basalt fibers or aramid fibers. It is considered that the hybridization of basalt / aramid fibers increases the bending strength. 
(2) Basalt fiber / aramid fiber It was found that the bending strength of high-hybrid composite material changes depending on the lamination pattern of two kinds of fibers. It is believed that this is caused by delamination caused by lamination of heterogeneous fibers, which is caused by a layer of weak fibers.

(3) The bending strength by the addition of HNT and the reinforcement effect in the ILSS test were confirmed. It is considered that HNT has a reinforcing effect because it acts as a bridge between fiber and resin.

(4) The fracture toughness, ILSS, and bending strength of specimens with HNT content of more than a certain level were decreased. This was confirmed by the SEM image as a result of HNT agglomeration due to the high surface energy of nanoparticle size HNT.

Acknowledgments: This research was supported by the Ministry of Trade, Industry \& Energy (MOTIE), Korea Institute for Advancement of Technology (KIAT) through the IDEA FACTORY Research Grant of N0001410.

\section{References}

1. Han, I.S.; Kim, S.Y.; Woo, S.K.; Hong, K.S.; Seo, D.W. Characteristics of Glass/Carbon Fiber Hybrid Composite Using by VARTM. Journal of the Korean Ceramic Society. 2006, 10, 607-612.

2. Kim, Y.H.; Lee, J.W.; Park, C.W.; Park, S.J. Thermal Characteristics of Hybrid Composites for Application to Surfboard. J. Ocean Eng. and Tech. 2014, 28, 351-355.

3. Kim, J.W. Effect of Temperature on Flexural Properties of Carbon-Basalt/Epoxy Interply Hybrid Composteis. Master's Thesis, Chonbuk National University, JeonJu, Korea, 2014.

4. Jeong, J.S.; Cheong, S.K. Mode II Interlamivar Fracture Toughness of Hybrid Composites Inserted with Different Types of Non-woven Tissues. Composite Research. 2013, 26, 141-145.

5. Lee, J.W. A Study on the Interfacial Properties of Nano Particles-contained Carbon Fiber Reinforced Composite Materials. Doctorate Thesis, Pusan National University, Busan, Korea, 2016.

6. Moon, Y.J.; Choi, J.Y.; Kim, B.A.; Moon, C.K. Effect of TiO2 Nanoparticle on the Mechanical and Thermal Properties of Epoxy Resin Composites. J. Korea Soc. Power Syst. Eng. 2010, 14, 68-75.

7. Oh, D.Y. Physical and Chemical Treatment to Improve Interfacial Adhesion Strength of Carbon Fiber Reinforced with Hybrid Composites. Master's Thesis, Pusan National University, Busan, Korea, 2012.

8. Hong, M.S.; Bae, K.M.; Choi, W.K.; Lee, H.S.; Park, S.J; An, K.H.; Kim, B.J. A Study on Mechanical Interfacial Properties of Copper-plated Carbon Fibers/Epoxy Resin Composites. Appl. Chem. Eng. 2012, 23, 313-319.

9. Kim, Y.H.; Park, S.J.; Lee, J.W.; Moon, K.M. A Study on the Effect of Halloysite Nanoparticle Addition on the Strength of Glass Fiber Reinforced Plastic. Modern Physics Leeters B 2015, 29, 1540003.

10. Lee, J.W.; Kim. Y.H.; Jung, M.K.; Yoon, S.W.; Park, J.M. Fabrication of Carbon/Basalt Hybrid Composites and Evaluation of Mechanical Properties. Composite Research. 2014, 27, 14-18. 